\title{
Baseline anti-NS4a antibodies in combination with on-treatment quantitative HCV-RNA reliably identifies non responders to peginterferon-ribavirin combination therapy after 4 weeks of treatment.
}

\author{
Hans Orlent ${ }^{1,2}$, I sabelle Desombere ${ }^{3}$, Bettina Hansen ${ }^{2,4}$, Hans Van Vlierberghe ${ }^{5}$, Bart \\ Haagmans $^{6}$, Robert J. De Knegt ${ }^{2}$, Solko W. Schalm², Geert Leroux-Roels ${ }^{3}$, Harry L.A. \\ Janssen² for the DITTO-HCV Study Group.
}

Short title: HCV week 4 stopping rule

\footnotetext{
Author Affiliation:

1: Department of Gastroenterology and Hepatology, AZ St. Jan AV, Bruges, Belgium.

2: Department of Gastroenterology and Hepatology, Erasmus MC University Medical Center, Rotterdam, Netherlands

3: Center for Vaccinology, Ghent University and Hospital, Ghent, Belgium

4: Department of Epidemiology and Biostatistics, Erasmus MC University Medical Center, Rotterdam, Netherlands

5: Department of Gastroenterology and Hepatology, University Hospital of Ghent, Ghent, Belgium.

6: Institute of Virology, Erasmus MC University Medical Center, Rotterdam, Netherlands
}

Address of correspondence:

Dr. H. Orlent

Department of Gastroenterology and Hepatology
AZ ST J an AV
Ruddershove 10
8000 Brugge, Belgium
Fax: +32.50 .452179$
E-mail: Hans.Orlent@azbrugge.be 


\section{Abstract.}

BACKGROUND: Early detection of non responders to hepatitis C therapy limits unnecessary exposure to treatment and its side effects. A recent algorithm combining baseline anti-NS4a antibodies and on treatment quantitative PCR identified non responders to a combination of interferon and ribavirin after 1 week of treatment. AI M: To validate a stopping rule based on baseline anti-NS4a antibody levels and early on treatment virological response in treatment naive genotype 1 chronic hepatitis $C$ patients treated with the current standard pegylated interferon and ribavirin combination therapy.

METHODS: Eighty-nine (89) genotype 1 patients from the DITTO study treated for 48 weeks with standard 180 Mg PEG-IFN- $\alpha-2 a$ weekly and ribavirin 1000-1200 mg daily, were analysed. Baseline anti-NS4a antibody ELISA (NS4a AA 1687-1718) was performed on pre-treatment serum. HCV-RNA was assessed at day $0,1,4,7,8,15$, 22,29 , week $6,7,8,10,12$ and 6 weekly thereafter until end of treatment. Multiple regression logistic analysis was performed.

RESULTS: Overall 54/89 (61\%) patients achieved sustained viral response. A baseline anti-NS4a antibody titre $<1 / 1250$ correlated with absence of favourable initial viral decline according to variable response types $(p=0.015)$. The optimal algorithm was developed using the combination of the absence of anti-NS4a Ab $(<1 / 1250)$ at baseline and the presence of a viral load $\geq 100.000 \mathrm{IU} / \mathrm{ml}$ at week 4 . This algorithm has a specificity of $43 \%$ and negative predictive value of $100 \%$ to detect non response to standard PEG-IFN- $\alpha-2 a$ and ribavirin therapy at week 4 of therapy (intention to treat 
analysis).

CONCLUSIONS: The decision to stop therapy in genotype 1 chronic hepatitis $C$ patients treated with PEG-IFN- $\alpha-2 a$ and ribavirin can be confidently made after 4 weeks of treatment based on the absence of baseline anti-NS4a Ab and a week 4 HCV-RNA above. $100.000 \mathrm{IU} / \mathrm{ml}$.

Keywords: hepatitis C; HCV-RNA; peginterferon alfa-2a; ribavirin; stopping rule; anti NS4a antibodies 


\section{Introduction.}

Pegylated interferon- $\alpha-2 a$ and ribavirin (RBV) combination therapy induces a sustained virological response (SVR) in only half of the genotype 1 infected chronic hepatitis C patients. ${ }^{1}$ Accurate early recognition of those patients who will not clear the HCV infection despite a full 48 week treatment course allows early treatment discontinuation limiting unnecessary exposure to potential side effects. The absence of a more than 2 log drop from baseline viral load after 12 weeks of treatment detects $31 \%$ of non responder patients with a negative predictive value (NPV) of $97 \% .^{1,2}$ A stopping rule that would identify more non responder patients earlier on during the treatment would limit further the side effects and costs of the treatment for these patients.

Recently Desombere et al. developed a stopping algorithm based on the absence or presence of baseline anti-NS4a antibodies (Ab) and early on treatment viral load that detects non response with a NPV of $100 \%$ in a group of patients treated with standard interferon and RBV combination therapy. ${ }^{3}$

Our aim was to develop a stopping rule with at least similar NPV and improved specificity in comparison to the current week 12 stopping rule, based on baseline antiNS4a serology status and early on treatment quantitative viral load in patients treated with peginterferon- $\alpha-2 a$ and RBV combination therapy.

\section{Materials and Methods.}

The Dynamically Individualized Treatment of hepatitis C Infection and Correlates of Viral/Host dynamics (DITTO) HCV study group recruited 270 chronic hepatitis C patients 
for an open label phase 3 international multicenter trial. ${ }^{4}$ Treatment-naive patients with compensated liver disease and the following characteristics were eligible: positive test for anti-HCV antibody, HCV-RNA > 1000 IU/ml (Cobas Amplicor HCV Monitor v. 2.0 assay, Roche Diagnostics, lower limit of detection 600 IU/ml), two serum alanine aminotransferase values above the upper limit of normal within 6 months of treatment initiation, and a pre-treatment (within previous 12 months) liver biopsy consistent with a diagnosis of chronic hepatitis C. The major exclusion criteria were previously reported. ${ }^{4}$ Patients were randomised after 6 weeks of combination therapy with peginterferon- $\alpha-2 a$ and weight based RBV combination therapy according to initial viral kinetics of the first 4 weeks to either individualised treatment or continued standard treatment until week 48. For this analysis the subset of 89 genotype 1 patients who completed at least 4 weeks of therapy in the standard treatment arm were analysed. All patients were treated with weekly injections of $180 \mu \mathrm{g}$ peginterferon- $\alpha-2 \mathrm{a}$ in combination with daily oral RBV at a dose of 1000 or $1200 \mathrm{mg}$ depending of bodyweight below or above $75 \mathrm{~kg}$. All patients were motivated to continue treatment for the 48 week treatment period. Treatment could be stopped at the discretion of the investigator if HCV-RNA was still positive after 24 weeks of treatment.

Labatory assessments. HCV-genotyping was confirmed centrally at Erasmus MC University Medical Center Rotterdam by reverse hybridization (Inno LipA HCV II; Innogenetics, Gent, Belgium). Baseline anti-NS4a ELISA was determined on a pretreatment serum sample (NS4a AA 1687-1718, horseradish peroxidise-labelled goat antihuman IgG antibody; dilutions $1 / 10,1 / 100,1 / 250,1 / 1250,1 / 16250$ ) in the CEVAC lab 
in Ghent as described previously. ${ }^{3}$ HCV-RNA was determined centrally in Rotterdam with the Cobas Amplicor HCV Monitor v. 2.0 assay (Roche Diagnostics, lower limit of detection $600 \mathrm{IU} / \mathrm{ml})$ at day $0,1,4,7,8,15,22,29$, week $6,7,8,10$ and 12 and 6 weekly thereafter until end of treatment. All samples that tested negative with the quantitative assay were retested with the Cobas Amplicor HCV Monitor v2.0 qualitative assay (Roche Diagnostics, lower limit of detection $50 \mathrm{lU} / \mathrm{ml}$ ).

Definition of response type: In the DITTO study Rapid Viral Response (RVR) was defined as a $\geq 2$ log drop of HCV-RNA during the first 4 weeks and a second-phase decline $\geq 0.09 \mathrm{log} /$ day. Slow partial response (SPR) was defined as no RVR but a second-phase decline $\geq 0.09 \log /$ day and (i) a $\geq 1 \log$ drop of HCV-RNA during the first 4 weeks or (ii) a $\geq 0.6$ log drop of HCV-RNA during the first 4 weeks and a first-phase decline of $\geq 0.5 \mathrm{log}$. SVR was defined as negative qualitative HCV-RNA $<50 \mathrm{IU} / \mathrm{ml}$ at 24 weeks after the end of treatment (week 72).

\section{Study end points and objectives.}

The primary objective of this study was to develop a stopping rule that reliably detects non responder patients during the first weeks of treatment based on the absence of pre-treatment anti NS4a Ab and the presence of a rapid on treatment HCV-RNA decline. Two items were considered of paramount importance in the development of the stopping rule: NPV and Specificity. The NPV of a stopping rule reflects the probability that the algorithm indeed predicts a true "non-response", or in other words how sure one can be that no potential SVR patients will be lost by applying the early stopping rule. The specificity (SPEC) depicts the fraction of all non-responding patients that will 
indeed be identified by the prediction criteria. The goal is the highest possible specificity with a NPV of $100 \%$. For this study we aimed to develop a stopping rule that could detect at least a similar proportion of non responders as the current week 122 log rule, but to detect these patients at an earlier stage during treatment while maintaining excellent NPV.

\section{Statistical analysis.}

Multiple logistic regression analysis was used to explore a possible correlation between non sustained virological response, baseline anti-NS4a Ab titre and viral load at baseline and day $0,1,4,7,8,15,22,29$. All analyses were performed as intention to treat analysis, categorizing patients with missing week 72 data as non sustained virological patients. Receiver Operating Characteristic curves and Akaike Information Criteria were used to investigate the non-inferiority of specificity and NPV of a possible new stopping algorithm with the current week 122 log rule specificity and NPV. All analyses were performed using SPSS ${ }^{\circledR} 12.0$ statistical software (SPSS Inc., Chicago, Illinois, US).

\section{Results.}

Patient characteristics. The study population $(n=89)$ consisted genotype 1 infected chronic hepatitis C patients with a mean age of 41 years (20-66), predominantly of Caucasian origin. Sixty-four percent of patients (57/89) had a high baseline viremia of $\geq 0.8 \times 10^{6} \mathrm{IU} / \mathrm{ml}$ and the median viremia at baseline in this study group was $1.38 \times 10^{6}$ IU/ml. Ten percent of patients had a baseline viremia $<10^{5} \mathrm{IU} / \mathrm{ml}$. Cirrhosis, defined as an Ishak score of 5 or 6 , was present in $10 \%$ of the patients (Table 1 ). SVR in this 
study group with a high baseline viremia was $61 \%(54 / 89)$ when treated with a standard 48 weeks peginterferon- $\alpha-2 a$ and RBV combination therapy (intention to treat analysis).. SVR was achieved in 39/47 (83\%) and 14/30 (47\%) of the patients showing RVR and SPR according to the DITTO response types, respectively. 35/89 patients had no SVR at week 72 . Of these $9 / 89(10 \%)$ discontinued treatment prematurely prior to week 48. 15/89 (17\%) did not achieve week 48 end of treatment virological response. Relapse occurred in the remaining $11 / 89(12 \%)$ at week 72.

Baseline anti-NS4a antibody levels. Logistic regression analysis showed a correlation between baseline anti-NS4a Ab levels and the initial viral kinetics. Patients with a baseline anti-NS4a Ab titre of $<1 / 1250$ displayed significantly fewer rapid viral responses or slow partial responses compared to patients with a baseline anti-NS4a Ab titre $\geq 1 / 1250$ ( $p=0.015$ Chi-square) (Fig. 1). There was no statistically significant correlation between baseline anti-NS4a Ab levels and HCV-RNA negativity at any time point of treatment or with the SVR status at end of follow-up.

HCV-RNA at Week 4. A single HCV-RNA value exceeding $10^{5} \mathrm{IU} / \mathrm{ml}$ at week 4 was superior in detecting non responding patients early on during treatment (sensitivity 96.3\%, specificity 48.6\%, PPV 74.3\%, NPV 89.5\%) than a 2 log HCV-RNA decline at this time point. This parameter however lacked adequate NPV prohibiting its clinical application as stopping rule, since therapy would be incorrectly stopped in $10.5 \%$ of patients. 
HCV-RNA at week 12. Evaluation of the current week 122 log stopping rule in this group showed a sensitivity of $100 \%$, a specificity of $31 \%$, a PPV of $69 \%$ and a NPV of $100 \%$, confirming previously reported values. ${ }^{1,2}$ In this group of 89 patients, a stopping rule based on the absence of a week $12 \mathrm{HCV}$-RNA below $1600 \mathrm{IU} / \mathrm{ml}$ had the best specificity of $43 \%$ while maintaining a NPV of $100 \%$ to predict non response.

\section{Algorithm based on early HCV-RNA determination and baseline anti-NS4a} antibody titer. Multiple logistic regression analysis with the variables baseline antiNS4a Ab titre, HCV-RNA level and treatment duration was performed (Fig.2). An algorithm could be developed based on a baseline anti-NS4a Ab titre of $<1 / 1250$ and the presence of a HCV-RNA $\geq 10^{5} \mathrm{IU} / \mathrm{ml}$ after 4 weeks of treatment. Patients who had week 4 HCV-RNA of $\geq 10^{5} \mathrm{IU} / \mathrm{ml}$ and a baseline anti-NS4a Ab titre of $<1 / 1250$ had no chance of achieving SVR with continued treatment. This algorithm had a sensitivity of $100 \%$, specificity of $43 \%$, PPV of $73 \%$ and NPV of $100 \%$ to detect non response to standard peginterferon- $\alpha-2 a$ RBV combination therapy after 4 weeks of therapy_ (intention to treat analysis). The performance of this stopping rule is similar in the group of $80 / 89$ patients with a baseline HCV-RNA $\geq 10^{5} \mathrm{IU} / \mathrm{ml}$ as all patients with a week 4 HCV-RNA $<10^{5} \mathrm{IU} / \mathrm{ml}$ are to continue therapy with this rule (sensitivity $100 \%$, specificity 43\%, PPV 69\%, NPV 100\%). Our week 4 stopping rule categorised 7 of the 9/89 drop out patients as week 4 responders. The week 4 algorithm has a sensitivity of $100 \%$, specificity of $50 \%$, PPV of $81 \%$ and NPV of $100 \%$ in the $80 / 89$ patients who 


\section{continued treatment until week 48. It is hence fair to assume that the drop-out rate did} not favour a positive bias towards the week 4 algorithm.

The week 4 rule identified at least a similar proportion of non sustained virological responders as the current week 122 log rule (specificity 43 vs 31 respectively, Receiver Operating Characteristic curves: Akaike Information Criteria 91.2 and 98.3 and Area Under the Curve 0.724 and 0.657 for the new week 4 and current week 12 algorithm respectively) and a NPV of $100 \%$ at an 8 week earlier time point._Analysis of concordant and discordant results showed that all adherent week 122 log non sustained virological responders were identified by the week 4 algorithm. Consecutive application of the new week 4 algorithm and by the current week 122 log rule would detect $51 \%$ of non responders (18/35) after 12 weeks of treatment in this study group (Fig.3). 


\section{Discussion.}

The aim of this study was to develop a clinical applicable stopping rule that would allow earlier detection of non response compared to the current week 122 log stopping rule while maintaining a reliable NPV and detecting at least a similar proportion of non responder patients. The combination of a low anti-NS4a Ab titer at baseline, correlating with a low likelihood of favourable initial viral kinetics, and HCV-RNA determination at week 4, constitutes a stopping rule with adequate NPV and specificity. This stopping rule detects at least a similar proportion of non responders as the week 122 log rule, but does so 8 weeks earlier.

Careful examination of the three variables used in this analysis, namely HCV-RNA level, treatment duration and baseline anti NS4a Ab titre, revealed the following trends. The earlier the time point, the better the specificity and PPV but the worse the sensitivity and NPV for a given HCV-RNA cut-off or log decline value. This translates into the clinical knowledge that the presence of early on treatment HCV-RNA negativity favours subsequent SVR but is not an absolute requirement since a number of patients who achieve HCV-RNA negativity later than week 4 still become sustained responders with continued treatment. This prevents the development of a clinically relevant stopping algorithm based on a single qualitative HCV-RNA level alone before week 12 . In this cohort $96.3 \%$ of responder patients had a week $4 \mathrm{HCV}-\mathrm{RNA}$ below $10^{5} \mathrm{IU} / \mathrm{ml}$, the cut-off put forward in Desombere's algorithm. ${ }^{3}$ In patients treated with pegylated interferon this cut-off has clinical relevance after 4 weeks treatment duration whereas in Desombere's study in patients treated with short acting standard interferon this cut-off 
value appeared to be useful already after one week of therapy. The NPV of $90 \%$ of the week $4 \mathrm{HCV}$-RNA cut-off of $10^{5} \mathrm{IU} / \mathrm{ml}$ is insufficient to be used as a reliable stopping rule. We were able to increase the NPV of an early on treatment qualitative HCV-RNA level by adding_a baseline parameter to our algorithm. Our findings corroborate those of Desombere et al. ${ }^{3}$ in that decreasing baseline anti-NS4a Ab levels correlate with the absence of a favourable initial viral response to peginterferon and RBV combination treatment. The mechanism underlying this association is unclear at present. It has recently been shown that the HCV NS3/4a protease interferes with the innate immune response against HCV infection by abrogation of the RIG1 and TLR3 pathway. ${ }^{5-7}$ It has also been shown that interferon non responders have high baseline Interferon Stimulated Genes status (ISGs) without any further increase upon interferon administration compared to interferon responders. ${ }^{8}$ We speculate that the counteractive interaction of the NS3/4a protease with the innate immune system and the ISGs response is abrogated in the presence of a high titer of anti-NS4a specific antibodies, resulting in both better innate response and more favourable viral response to exogenous administered interferon.

Many baseline factors have been described that correlate with a lower probability to obtain SVR in genotype 1 patients with peginterferon and RBV treatment. Amongst these high viral load, advanced fibrotic stage, afro-american descendance, high BMI and presence of steatosis are recognised baseline factors that make SVR less likely. ${ }^{9}$, 10 Low IP10 interferon gamma inducible protein ${ }^{11,12}$, baseline homozygosity for the promoter diplopy of ACC-IL10 in non-hispanic caucasian patients ${ }^{13}$, and IL28B CC genotype 
regardless of descendance ${ }^{14,15}$ correlate with a higher probability to achieve SVR in adherent patients. The presence of baseline factors statistically in disfavour for SVR does not obviate the possibility to achieve SVR in a given patient completely. Withholding therapy on the basis of any of these parameters is thus unjustified and the need for early accurate on treatment detection of non response remains of paramount importance. Our new week 4 stopping rule is based on the presence of a baseline factor that correlates with favourable initial viral kinetics on the one hand, baseline level of anti-NS4a Ab, and on treatment HCV-RNA on the other hand as direct reflection of interferon response and indirect reflection of patient adherence. Our new paradigm will be easy to apply in current practice since week 4 HCV-RNA determinations have become custom management in most centres. This algorithm has a higher specificity and hence detects a larger proportion of non responding patients early on during treatment compared to a recently proposed stopping rule that is based on the absence of a 2 log viral decline and week $4 \mathrm{HCV}-\mathrm{RNA}>5.5 \mathrm{log} \mathrm{IU} / \mathrm{ml}^{16}$. It is important that the new, week 4 stopping rule has a $100 \%$ NPV and will thus stop therapy only in true non responders. All patients in this study were offered treatment for 48 weeks of treatment, irrespective of the week $12 \mathrm{HCV}$-RNA results, with the possibility to stop treatment if week $24 \mathrm{HCV}$ RNA was positive. The new stopping rule is therefore valid for the true occurrence of SVR or no SVR.

Our findings are based on data of a moderate size group and ideally are to be validated in the framework of larger patient groups. It is likely that our stopping rule will need adaptations with the advent of new future direct antiviral agent treatment regimes, 
since the majority of genotype 1 patients treated with these regimes have undetectable HCV-RNA after 4 weeks of combination therapies with these new agents. ${ }^{17,} 18$ In conclusion, a baseline anti-NS4a Ab titer of $<1 / 1250$ in combination with a week 4 PCR $\geq 10^{5} \mathrm{IU} / \mathrm{ml}$ reliably detects non response to 48 weeks of peginterferon and RBV therapy with a negative predictive value of $100 \%$ and does so 8 weeks earlier than the current week 122 log rule. 


\section{Appendix:}

Steering Committee members of the DITTO-HCV Study are: Carlo Ferrari (Divisione di Malattie Infettive, Azienda Ospedaliera di Parma, Parma, Italy), Avidan U. Neumann (Faculty of Life Sciences, Bar-Ilan UniversityRamat-Gan , Israel), J ean-Michel Pawlotsky (Department of Bacteriology and Virology, Hopital Henri Mondor - Université Paris XII, Creteil, France), Solko W. Schalm and Stefan Zeuzem (Medizinische Klinik II, Klinikum der J ohann Wolfgang Goethe-Universitat, Frankfurt A.M., Germany). 


\section{Tables.}

\section{Table 1: Patient characteristics.}

\begin{tabular}{|l|c|}
\hline Male/Female (\%) & $70 / 30$ \\
\hline Mean age in years (range) & $41(20-66)$ \\
\hline Caucasian (\%) & 97 \\
\hline Mean baseline ALT (xULN) & 2.9 \\
\hline Median HCV-RNA (IU/ml) & 1.380 .000 \\
\hline \# of pts with HCV-RNA >800.000 IU/ml & $57 / 89(64 \%)$ \\
\hline \# of pts with HCV-RNA <100.000 IU/ml & $9 / 89(10 \%)$ \\
\hline \# of pts with Ishak fibrosis stage 5-6 & $7 / 77(9 \%)$ \\
\hline \# of pts with SVR & $54 / 89(61 \%)$ \\
\hline
\end{tabular}




\section{Figure Legends.}

\section{Fiqure 1: Baseline anti-NS4a antibody titer (1/n) and early on treatment}

\section{response type.}

A baseline anti-NS4a Ab titer $\geq 1 / 1250$ correlates with the occurrence of more RVR/SPR versus absence of RVR/SPR in patients with a baseline anti-NS4a Ab $<1 / 1250$ (RVR: Rapid Viral Respnose, SPR Slow Partial Response) $(p=0.015)$.

\section{Figure 2: Multiple logistic regression analysis.}

Negative Predictive Value and Specificity of a new algorithm combining early PCR and baseline anti-NS4a serology: the addition of ascending baseline anti-NS4a antibody titer $<1 / 1250$ to a PCR cut-off of $100.000 \mathrm{IU} / \mathrm{ml}$ at week 4 creates a stopping rule with a specificity of $43 \%$ and a NPV of $100 \%$ (ITT analysis).

\section{Figure 3: Week 4 stopping rule:}

Patients with a baseline anti-NS4a antibody titer $<1 / 1250$ and a week 4 viremia $\geq 100.000$ IU/ml have no chance of obtaining SVR with continued treatment and can be advised to stop therapy. 
Figure 1: Baseline anti-NS4a antibody titer and early on treatment response type.

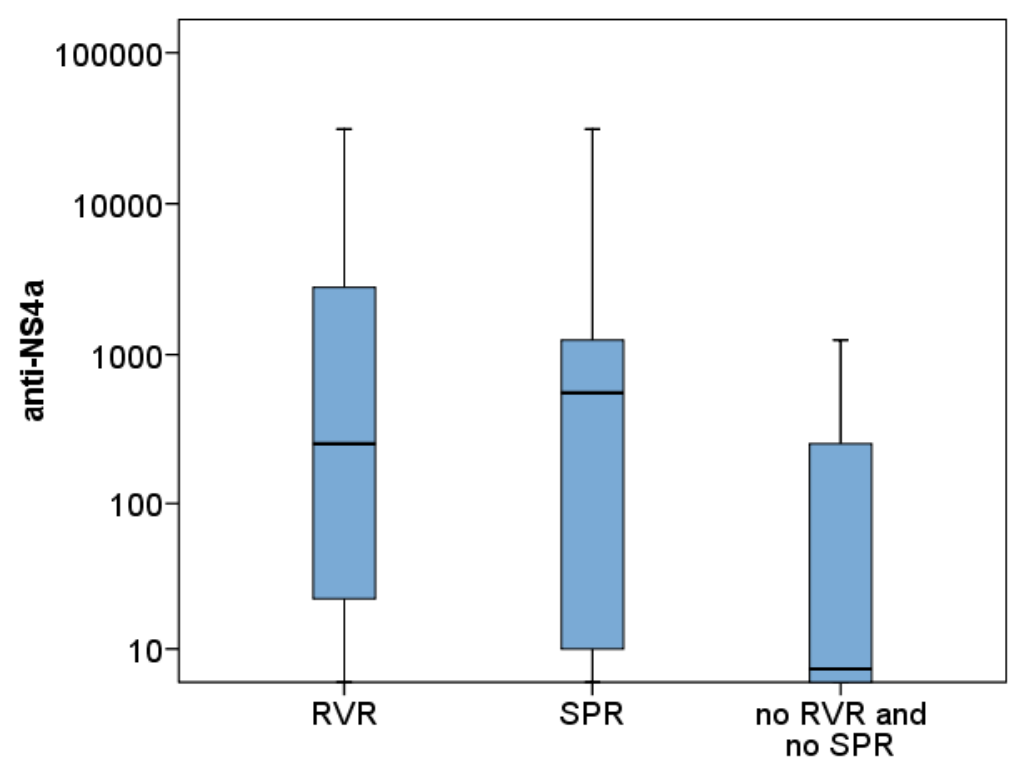


Figure 2: Multiple logistic regression analysis.

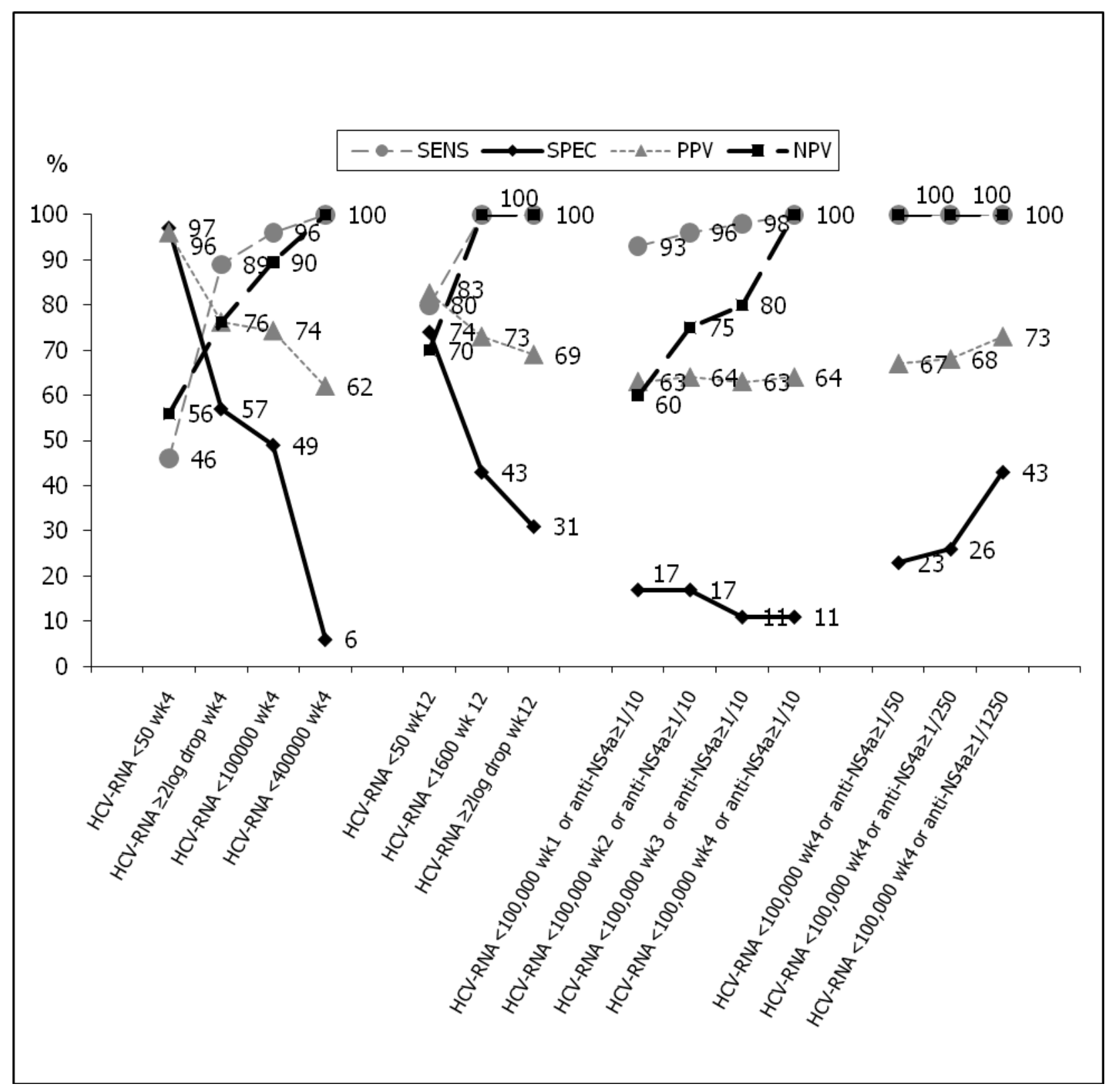


Figure 3: Week 4 stopping rule. 


\section{References}

1. Fried MW, Shiffman ML, Reddy KR, et al. Peginterferon Alfa-2a plus Ribavirin for Chronic Hepatitis C Virus Infection. N Engl J Med 2002;347(13):975-82.

2. Lee S, Heathcote $\mathrm{E}$, Reddy $\mathrm{K}$, et al. Prognostic factors and early predictability of sustained viral response with peginterferon alfa-2a (40KD). J Hepatol 2002;37(4):500.

3. Desombere I, Van Vlierberghe $\mathrm{H}$, Weiland O, et al. Serum levels of anti-NS4a and anti-NS5a predict treatment response of patients with chronic hepatitis C. J Med Virol 2007; 79(6):701-13.

4. Zeuzem S, Pawlotsky J M, Lukasiewicz E, et al. International, multicenter, randomized, controlled study comparing dynamically individualized versus standard treatment in patients with chronic hepatitis C. J Hepatol 2005;43(2):250-7.

5. Li XD, Sun L, Seth RB, Pineda G, Chen ZJ. Hepatitis C virus protease NS3/4A cleaves mitochondrial antiviral signaling protein off the mitochondria to evade innate immunity. Proc Natl Acad Sci U S A 2005; 102(49): 17717-22.

6. Li K, Foy E, Ferreon JC, et al. Immune evasion by hepatitis C virus NS3/4A protease-mediated cleavage of the Toll-like receptor 3 adaptor protein TRIF. Proc Natl Acad Sci U S A 2005; 102(8):2992-7.

7. Meylan E, Curran J, Hofmann K, et al. Cardif is an adaptor protein in the RIG-I antiviral pathway and is targeted by hepatitis C virus. Nature 2005;437(7062):1167-72.

8. Sarasin-Filipowic M, Oakeley EJ, Duong FH, et al. Interferon signaling and treatment outcome in chronic hepatitis C. Proc Natl Acad Sci U S A 2008;105(19): 7034-9.

9. Kau A, Vermehren J, Sarrazin C. Treatment predictors of a sustained virologic response in hepatitis B and C. J Hepatol 2008;49(4):634-51.

10. Ghany MG, Strader DB, Thomas DL, Seeff LB. Diagnosis, management, and treatment of hepatitis C: an update. Hepatology 2009;49(4):1335-74.

11. Lagging M, Romero Al, Westin J, et al. IP-10 predicts viral response and therapeutic outcome in difficult-to-treat patients with HCV genotype 1 infection. Hepatology 2006; 44(6):1617-25.

12. Romero Al, Lagging M, Westin J, et al. Interferon (IFN)-gamma-inducible protein-10: association with histological results, viral kinetics, and outcome during treatment with pegylated IFN-alpha $2 a$ and 
ribavirin for chronic hepatitis C virus infection. J Infect Dis 2006; 194(7):895-903.

13. Morgan TR, Lambrecht RW, Bonkovsky HL, et al. DNA polymorphisms and response to treatment in patients with chronic hepatitis C: results from the HALT-C trial. J Hepatol 2008;49(4):548-56.

14. Ge D, Fellay J, Thompson AJ, et al. Genetic variation in IL28B predicts hepatitis C treatmentinduced viral clearance. Nature 2009;461(7262):399-401.

15. Thomas DL, Thio CL, Martin MP, et al. Genetic variation in IL28B and spontaneous clearance of hepatitis C virus. Nature 2009;461(7265): 798-801.

16. Neumann AU, Pianko S, Zeuzem S, et al. Positive and negative prediction of sustained virologic response at weeks 2 and 4 of treatment with albinterferon alfa-2b or peginterferon alfa-2a in treatmentnaive patients with genotype 1, chronic hepatitis C. J Hepatol 2009;51(1):21-8.

17. McHutchison J G, Everson GT, Gordon SC, et al. Telaprevir with peginterferon and ribavirin for chronic HCV genotype 1 infection. N Engl J Med 2009;360(18):1827-38.

18. Hezode C, Forestier N, Dusheiko G, et al. Telaprevir and peginterferon with or without ribavirin for chronic HCV infection. N Engl J Med 2009;360(18):1839-50. 